\title{
The Extended Inverse Weibull Distribution: Properties and Applications
}

\author{
Said Alkarni $\mathbb{D}^{1},{ }^{1}$ Ahmed Z. Afify, ${ }^{2}$ I. Elbatal, ${ }^{3}$ and M. Elgarhy ${ }^{4}$ \\ ${ }^{1}$ Department of Quantitative Analysis, King Saud University, Riyadh, Saudi Arabia \\ ${ }^{2}$ Department of Statistics, Mathematics and Insurance, BenhaUniversity, Banha, Egypt \\ ${ }^{3}$ Department of Mathematics and Statistics, College of Science, Imam Mohammad Ibn Saud Islamic University (IMSIU), \\ Riyadh, Saudi Arabia \\ ${ }^{4}$ Valley High Institute for Management Finance and Information Systems, Obour, Qaliubia, Egypt
}

Correspondence should be addressed to Said Alkarni; salkarni@ksu.edu.sa

Received 12 April 2020; Revised 14 August 2020; Accepted 19 September 2020; Published 20 October 2020

Academic Editor: Hassan Zargarzadeh

Copyright (C) 2020 Said Alkarni et al. This is an open access article distributed under the Creative Commons Attribution License, which permits unrestricted use, distribution, and reproduction in any medium, provided the original work is properly cited.

This paper proposes the new three-parameter type I half-logistic inverse Weibull (TIHLIW) distribution which generalizes the inverse Weibull model. The density function of the TIHLIW can be expressed as a linear combination of the inverse Weibull densities. Some mathematical quantities of the proposed TIHLIW model are derived. Four estimation methods, namely, the maximum likelihood, least squares, weighted least squares, and Cramér-von Mises methods, are utilized to estimate the TIHLIW parameters. Simulation results are presented to assess the performance of the proposed estimation methods. The importance of the TIHLIW model is studied via a real data application.

\section{Introduction}

The inverse Weibull (IW) distribution is also known as reciprocal Weibull distribution (see [1, 2]). Keller et al. [3] used the IW distribution to describe the degradation phenomena of mechanical components such as crankshaft and pistons of diesel engines. Further, the IW model has many important applications in reliability engineering, infant mortality, useful life, wear-out periods, life testing, and service records (see [4]).

The cumulative distribution function $(\mathrm{CDF})$ of the IW model is

$$
G_{\mathrm{IW}}(x ; \alpha, \beta)=e^{-\alpha x^{-\beta}}, \quad x>0, \alpha, \beta>0 .
$$

Its associated probability density function (PDF) has the following form:

$$
g_{\mathrm{IW}}(x ; \alpha, \beta)=\alpha \beta x^{-\beta-1} e^{-\alpha x^{-\beta}}, \quad x>0, \alpha, \beta>0 .
$$

The statistical literature contains several extensions of the IW model, see, for example, beta IW by Khan [5], generalized IW by de Gusmão et al. [6], modified IW by
Khan and King [4], gamma IW by Pararai et al. [7], Kumaraswamy generalized IW by Oluyede and Yang [8], Kumaraswamy modified IW by Aryal and Elbatal [9], Marshall-Olkin IW by Okasha et al. [10], and alpha power IW by Basheer [11].

Many generalized classes of distributions have been proposed for modeling real-life data in several applied fields such as reliability, engineering, biological studies, economics, medical sciences, environmental sciences, and finance. For example, Marshall and Olkin [12] proposed Marshall-Olkin-G, Shaw and Buckley [13] defined transmuted-G, Cordeiro and de Castro [14] pioneered Kumaraswamy-G, Alizadeh et al. [15] proposed the extended odd Weibull-G, and Cordeiro et al. [16] studied the odd LomaxG family.

Cordeiro et al. [17] proposed the type I half-logistic-G (TIHL-G) class. The CDF of the TIHL-G family of distributions is given (for $x \in \mathbb{R}$ ) by

$$
F(x ; \lambda, \delta)=\frac{1-[1-G(x ; \delta)]^{\lambda}}{1+[1-G(x ; \delta)]^{\lambda}}, \quad \lambda>0,
$$


where $G(x ; \delta)$ refers to the baseline CDF with a parameter vector $\delta$. The CDF in (3) is a wider class which can be used to generate more flexible extended distributions.

The associated PDF of (3) has the form

$$
f(x ; \lambda, \delta)=\frac{2 \lambda g(x ; \delta)[1-G(x ; \delta)]^{\lambda-1}}{\left\{1+[1-G(x ; \delta)]^{\lambda}\right\}^{2}},
$$

where $g(x ; \delta)$ is the baseline PDF. The hazard rate function (HRF) of the TIHL-G family is

$$
h(x, \lambda, \delta)=\frac{\lambda g(x ; \delta)}{[1-G(x ; \delta)]\left\{1+[1-G(x ; \delta)]^{\lambda}\right\}} .
$$

In this paper, we propose a new lifetime model called the type I half-logistic inverse Weibull (TIHLIW) model. The proposed model can be used, as a good alternative to some existing distributions, in modeling several real data.

The paper is outlined as follows. In Section 2, we define the TIHLIW distribution and derive a useful representation for its PDF. The mathematical properties of the TIHLIW distribution are derived in Section 3. In Section 4, the TIHLIW parameters are estimated via four methods, namely, the maximum likelihood, least squares, weighted least squares, and Cramér-von Mises estimators. These estimators are compared via some simulations in Section 5 . In Section 6, we illustrate the flexibility and potentiality of the TIHLIW model using a real data set. Finally, some concluding remarks are offered in Section 7.

\section{The TIHLIW Distribution}

The CDF of the three-parameter TIHLIW distribution follows, by replacing equation (1) in (3), as

$$
F(x ; \lambda, \alpha, \beta)=\frac{1-\left(1-e^{-\alpha x^{-\beta}}\right)^{\lambda}}{1+\left(1-e^{-\alpha x^{-\beta}}\right)^{\lambda}}
$$

The corresponding PDF of (4) reduces to

$$
f(x ; \lambda, \alpha, \beta)=\frac{2 \lambda \alpha \beta\left(x^{-\beta-1}\right)\left(e^{-\alpha x^{-\beta}}\right)\left(1-e^{-\alpha x^{-\beta}}\right)^{\lambda-1}}{\left[1+\left(1-e^{-\alpha x^{-\beta}}\right)^{\lambda}\right]^{2}} \text {. }
$$

The HRF of the TIHLIW distribution takes the form

$$
h(x ; \lambda, \alpha, \beta)=\frac{\lambda \alpha \beta\left(x^{-\beta-1}\right)\left(e^{-\alpha x^{-\beta}}\right)}{\left(1-e^{-\alpha x^{-\beta}}\right)\left[1+\left(1-e^{-\alpha x^{-\beta}}\right)^{\lambda}\right]} .
$$

Figure 1 provides some shapes of the PDF and HRF of the TIHLIW distribution for some different values of the parameters.

The TIHLIW distribution is a very flexible model that approaches to different distributions as special submodels:

(1) If $\alpha=1$, then the TIHLIW distribution reduces to the type I half-logistic Fréchet (TIHLFr) distribution

(2) If $\beta=1$, TIHLIW distribution reduces to the type I half-logistic inverse exponential (TIHLIE) distribution

(3) If $\beta=2$, we have the type I half-logistic inverse Rayleigh (TIHLIR) distribution

2.1. Linear Representation. In this section, we express the TIHLIW PDF as a mixture linear representation of IW densities.

Consider the power series:

$$
(1+z)^{-k}=\sum_{n=0}^{\infty}\left(\begin{array}{c}
-k \\
n
\end{array}\right) z^{n}, \quad|z|<1 \text { and } k>0 .
$$

Expanding $\left[1+\left(1-e^{-\alpha x^{-\beta}}\right)^{\lambda}\right]^{-2}$ using (9), we can write (7) as

$$
f(x ; \lambda, \alpha, \beta)=2 \lambda \alpha \beta\left(x^{-\beta-1}\right)\left(e^{-\alpha x^{-\beta}}\right) \sum_{i=0}^{\infty}\left(\begin{array}{c}
-2 \\
i
\end{array}\right)\left(1-e^{-\alpha x^{-\beta}}\right)^{\lambda(i+1)-1} .
$$

Consider the power series:

$$
(1-z)^{b-1}=\sum_{j=0}^{\infty}(-1)^{j}\left(\begin{array}{c}
b-1 \\
j
\end{array}\right) z^{j}, \quad|z|<1 \text { and } b>0 .
$$

Using the power series (11) and after some algebra, the TIHLIW PDF reduces to

$$
\begin{aligned}
f(x ; \lambda, \alpha, \beta) & =2 \lambda \alpha \beta\left(x^{-\beta-1}\right) \sum_{i, j=0}^{\infty}(-1)^{j}\left(\begin{array}{c}
-2 \\
i
\end{array}\right)\left(\begin{array}{c}
\lambda(i+1)-1 \\
j
\end{array}\right)\left(e^{-\alpha(j+1) x^{-\beta}}\right) \\
& =\sum_{j=0}^{\infty} \varphi_{j} g(x ; \alpha(j+1), \beta),
\end{aligned}
$$

where $g(x ; \alpha(j+1), \beta)$ refers to the IW PDF with parameters $\alpha(j+1)$ and $\beta$, and

$$
\varphi_{j}=2 \lambda \sum_{i=0}^{\infty} \frac{(-1)^{j}}{(j+1)}\left(\begin{array}{c}
-2 \\
i
\end{array}\right)\left(\begin{array}{c}
\lambda(i+1)-1 \\
j
\end{array}\right) .
$$



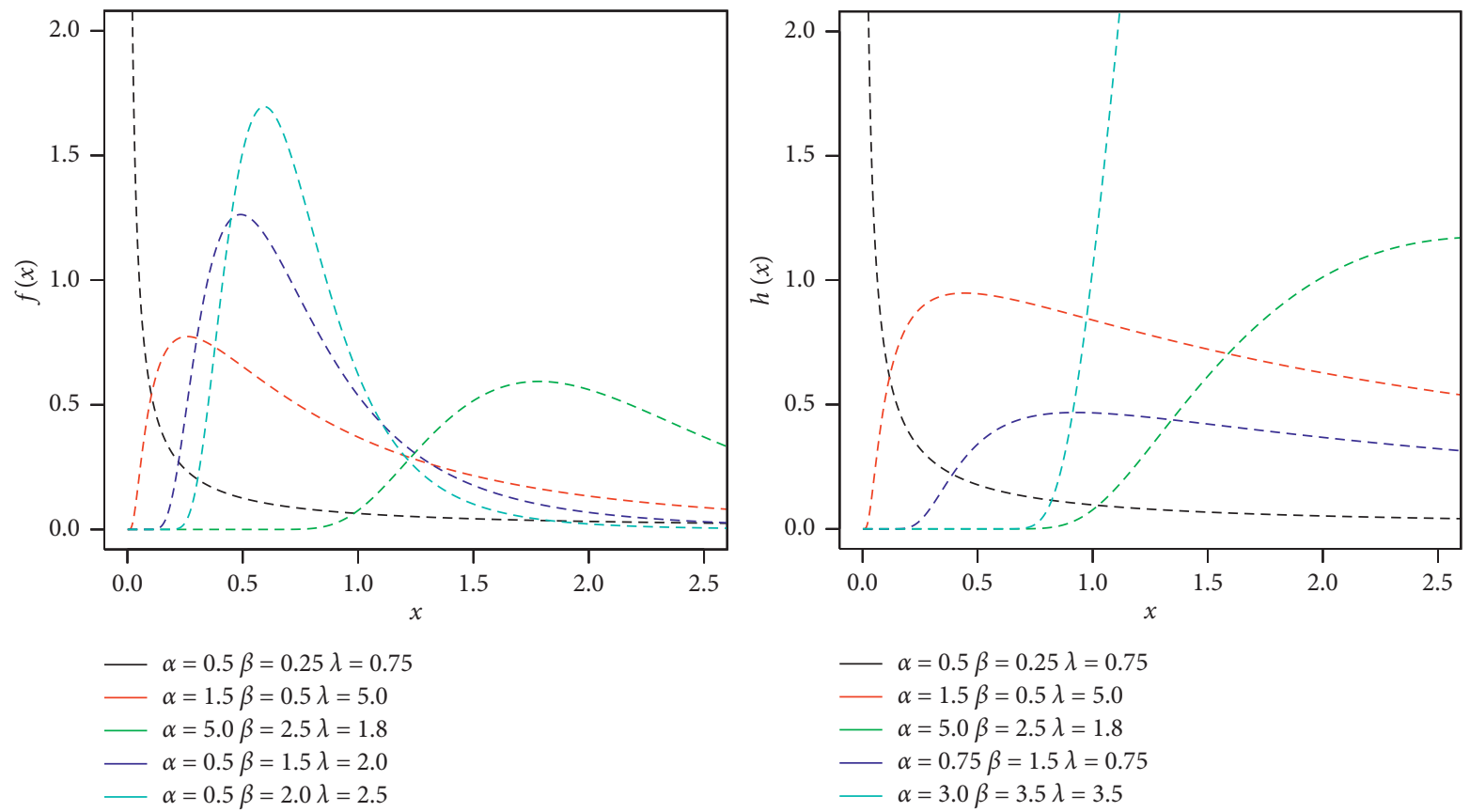

FIgURE 1: Plots of the PDF and HRF of the TIHLIW distribution for different values of parameters.

Equation (12) means that the PDF of the TIHLIW is a linear combination of the IW densities and can be used to calculate some mathematical quantities of the TIHLIW model from those of the IW distribution.

Consider the random variable $(\mathrm{RV}) Y \sim \operatorname{IW}(\alpha, \beta)$ in $(1)$. For $n<\beta$, the $n$th ordinary and incomplete moments of $Y$ are given by

$$
\begin{gathered}
\mu_{n, Y}^{\prime}=\alpha^{(n / \beta)} \Gamma\left(1-\frac{n}{\beta}\right), \\
\varphi_{n, Y}(t)=\alpha^{(n / \beta)} \gamma\left(1-\frac{n}{\beta}, \alpha t^{\beta}\right),
\end{gathered}
$$

respectively, where $\Gamma(b)=\int_{0}^{\infty} w^{b-1} e^{-w} \mathrm{~d} w$ is the complete gamma function and $\gamma(b, z)=\int_{0}^{z} w^{b-1} e^{-w} \mathrm{~d} w$ is the lower incomplete gamma function.

\section{Some Properties}

In this section, we studied some statistical properties of the TIHLIW distribution, such as quantile function, ordinary moments, moment generating function, incomplete moment, and mean deviation.

3.1. Quantile and Moment-Generating Functions. As RV X has CDF of TIHLIW distribution, the quantile function $(\mathrm{QF})$ is defined by

$$
Q(u)=\inf \{x \in R: F(x) \geq u\},
$$

where $(0<u<1)$. This relation is used to find the QF of TIHLIW distribution as follows:

$$
Q(u)=\left\{\frac{-1}{\alpha} \ln \left[1-\left(\frac{1-u}{1+u}\right)^{\lambda}\right]\right\}^{(-1 / \beta)}, \quad u \in(0,1) .
$$

The above equation can be used to generate TIHLIW random variates. Here, we obtain the MGF of the IW distribution (1) by setting $w=x^{-1}$ :

$$
M(t ; \alpha, \beta)=\alpha \beta \int_{0}^{\infty} \exp \left(\frac{t}{w}\right) w^{\beta-1} \exp \left(-\alpha w^{\beta}\right) \mathrm{d} w
$$

By expanding $\exp (t / w)$ and calculating the integral, we can write

$$
\begin{aligned}
M(t ; \alpha, \beta) & =\alpha \beta \int_{0}^{\infty} \sum_{n=0}^{\infty} \frac{t^{n}}{n !}\left(w^{\beta-n-1}\right) \exp \left(-\alpha w^{\beta}\right) \mathrm{d} w \\
& =\sum_{n=0}^{\infty} \frac{\alpha^{(n / \beta)} t^{n}}{n !} \Gamma\left(\frac{\beta-n}{\beta}\right) .
\end{aligned}
$$

Using the Wright generalized hypergeometric function [18],

${ }_{p} \psi_{q}\left[\begin{array}{l}\left(\alpha_{1}, C_{1}\right), \ldots,\left(\alpha_{p}, C_{p}\right) \\ \left(\beta_{1}, D_{1}\right), \ldots,\left(\beta_{q}, D_{q}\right)\end{array} ; x\right]=\sum_{m=0}^{\infty} \frac{\prod_{i=1}^{p} \Gamma\left(\alpha_{i}+C_{i} m\right)}{\prod_{i=1}^{q} \Gamma\left(\beta_{i}+D_{i} m\right)} \frac{x^{m}}{m !}$.

The MGF of the IW distribution has the form

$$
M(t ; \alpha, \beta)={ }_{1} \psi_{0}\left[\begin{array}{c}
\left(1,-\beta^{-1}\right) \\
-
\end{array} \alpha^{(1 / \beta)} t\right] .
$$

Using equations (12) and (20), the MGF of the TIHLIW distribution reduces to 


$$
M(t)=\sum_{j=0}^{\infty} \varphi_{j 1} \psi_{0}\left[\begin{array}{c}
\left(1,-\beta^{-1}\right) \\
-
\end{array}[\alpha(j+1)]^{(1 / \beta)} t\right] .
$$

3.2. Moments. The sth ordinary moment of RV X is

$$
\mu_{s}^{\prime}=E\left(X^{s}\right)=\sum_{j=0}^{\infty} \varphi_{j} \int_{0}^{\infty} x^{s} g(x ; \alpha(j+1)), \beta \mathrm{d} x .
$$

For $(s<\beta)$, we obtain

$$
\mu_{s}^{\prime}=\sum_{j=0}^{\infty} \varphi_{j}[\alpha(j+1)]^{(s / \beta)} \Gamma\left(1-\frac{s}{\beta}\right) .
$$

Setting $(s=1)$ in $(23)$, we obtain the mean of $X$.

The sth incomplete moment of the TIHLIW distribution is defined by $\varphi_{s}(t)=\int_{0}^{t} x^{s} f(x) \mathrm{d} x$.

Using equation (12), we can write

$$
\varphi_{s}(t)=\sum_{j=0}^{\infty} \varphi_{j} \int_{0}^{t} x^{s} g(x ; \alpha(j+1), \beta) .
$$

Hence, we obtain the sth incomplete moment of the TIHLIW distribution:

$$
\varphi_{s}(t)=\sum_{j=0}^{\infty} \varphi_{j}[\alpha(j+1)]^{(s / \beta)} \gamma\left(1-\frac{s}{\beta}, \alpha(j+1) t^{\beta}\right), \quad s<\beta .
$$

The first incomplete moment which follows by setting $(s=1)$ in the above equation is

$$
\varphi_{1}(t)=\sum_{j=0}^{\infty} \varphi_{j}[\alpha(j+1)]^{(1 / \beta)} \gamma\left(1-\frac{1}{\beta}, \alpha(j+1) t^{\beta}\right), \quad \beta>1 .
$$

3.3. Mean Residual Life and Mean Waiting Time. The mean residual life (MRL) has useful applications in economics, life insurance, biomedical sciences, demography, product quality control, and product technology (see [19]). The MRL refers to the expected additional life length for a unit that is alive at age $t$, and it is defined by $m_{X}(t)=E(X-t \mid X>t)$, $t>0$.

The MRL of $X$ can be calculated by the formula:

$$
m_{X}(t)=\frac{\left[1-\varphi_{1}(t)\right]}{S(t)-t},
$$

where $S(t)$ is the survival function of $X$.

By inserting (26) in (27), the MRL of the TIHLIW distribution follows as

$$
m_{X}(t)=\frac{1}{S(t)} \sum_{j=0}^{\infty} \varphi_{j}[\alpha(j+1)]^{(1 / \beta)} \gamma\left(1-\frac{1}{\beta}, \alpha(j+1) t^{\beta}\right)-t .
$$

The mean inactivity time (MIT) (mean waiting time) is defined by $M_{X}(t)=E(t-X \mid X \leq t), t>0$, and it can be calculated by the formula:

$$
M_{X}(t)=t-\left[\frac{\varphi_{1}(t)}{F(t)}\right] .
$$

By substituting (26) in (29), the MIT of the TIHLIW distribution follows as

$$
M_{X}(t)=t-\frac{1}{F(t)} \sum_{j=0}^{\infty} \varphi_{j}[\alpha(j+1)]^{(1 / \beta)} \gamma\left(1-\frac{1}{\beta}, \alpha(j+1) t^{\beta}\right) \text {. }
$$

3.4. Order Statistics. Consider the random sample from the TIHLIW $(\lambda, \alpha, \beta)$ denoted by $\left(X_{1}, \ldots, X_{n}\right)$ and its associated order statistics denoted by $\left(X_{(1)}, \ldots, X_{(n)}\right)$. The PDF of the $r$ th order statistic is denoted by $\left(X_{(r)}, 1 \leq r \leq n\right)$ and can be expressed as

$$
f_{(r)}(x)=\frac{n !}{(r-1) !(n-r) !} \frac{2 \lambda \alpha \beta\left(x^{-\beta-1}\right)\left(e^{-\alpha x^{-\beta}}\right)\left(1-e^{-\alpha x^{-\beta}}\right)^{\lambda-1}}{\left[1+\left(1-e^{-\alpha x^{-\beta}}\right)^{\lambda}\right]^{2}} \times\left[\frac{1-\left(1-e^{-\alpha x^{-\beta}}\right)^{\lambda}}{1+\left(1-e^{-\alpha x^{-\beta}}\right)^{\lambda}}\right]^{r-1}\left[\frac{2\left(1-e^{-\alpha x^{-\beta}}\right)^{\lambda}}{1+\left(1-e^{-\alpha x^{-\beta}}\right)^{\lambda}}\right]^{n-r}
$$

The PDF of $X_{(r)}$ is defined also by the formula:

$$
f_{(r)}(x)=\frac{f(x)}{B(r, n-r+1)} \sum_{j=0}^{n-r}(-1)^{j}\left(\begin{array}{c}
n-r \\
j
\end{array}\right) F^{j+r-1}(x) .
$$

Using the PDF and CDF of the TIHLIW distribution, equation (32) reduces to 


$$
f(x) F^{j+r-1}(x)=\frac{2 \lambda \alpha \beta\left(x^{-\beta-1}\right)\left(e^{-\alpha x^{-\beta}}\right)\left(1-e^{-\alpha x^{-\beta}}\right)^{\lambda-1}}{\left[1+\left(1-e^{-\alpha x^{-\beta}}\right)^{\lambda}\right]^{j+r+1}}\left[1-\left(1-e^{-\alpha x^{-\beta}}\right)^{\lambda}\right]^{j+r-1} .
$$

Using expansion (9) and the power series,

We can write

$(1-z)^{b-1}=\sum_{j=0}^{b-1}(-1)^{j}\left(\begin{array}{c}b-1 \\ j\end{array}\right) z^{j}, \quad b$ positive integer.

$$
f(x) F^{j+r-1}(x)=2 \lambda \alpha \beta\left(x^{-\beta-1}\right)\left(e^{-\alpha x^{-\beta}}\right) \sum_{l=0}^{\infty} \sum_{i=0}^{j+r-1}(-1)^{i}\left(\begin{array}{c}
-j-r-1 \\
l
\end{array}\right) \times\left(\begin{array}{c}
j+r-1 \\
i
\end{array}\right)\left(1-e^{-\alpha x^{-\beta}}\right)^{\lambda(l+i+1)-1} .
$$

Applying the power series (11), equation (35) reduces to

$$
f(x) F^{j+r-1}(x)=2 \lambda \alpha \beta\left(x^{-\beta-1}\right) \sum_{l=0}^{\infty} \sum_{i=0}^{j+r-1}(-1)^{i}\left(\begin{array}{c}
-j-r-1 \\
l
\end{array}\right)\left(\begin{array}{c}
j+r-1 \\
i
\end{array}\right) \times\left(\begin{array}{c}
\lambda(l+i+1)-1 \\
k
\end{array}\right) e^{-\alpha(k+1) x^{-\beta}}
$$

By replacing (36) in equation (32), we obtain

$f_{(r)}(x)=\frac{2 \lambda}{\mathrm{B}(r, n-r+1)} \sum_{j=0}^{n-r} \sum_{l, k=0}^{\infty} \sum_{i=0}^{j+r-1}(-1)^{j+i+k}\left(\begin{array}{c}n-r \\ j\end{array}\right)\left(\begin{array}{c}-j-r-1 \\ l\end{array}\right) \times\left(\begin{array}{c}j+r-1 \\ i\end{array}\right)\left(\begin{array}{c}\lambda(l+i+1)-1 \\ k\end{array}\right) \alpha \beta x^{-\beta-1} e^{-\alpha(k+1) x^{-\beta}}$

Hence, we can write

$$
f_{(r)}(x)=\sum_{k=0}^{\infty} b_{k} g(x ; \alpha(k+1), \beta)
$$

where $g(x ; \alpha(k+1), \beta)$ denotes the IW PDF with parameters $\beta$ and $\alpha(k+1)$, and

$$
b_{k}=\sum_{j=0}^{n-r} \sum_{l=0}^{\infty} \sum_{i=0}^{j+r-1} \frac{2 \lambda(-1)^{j+i+k}}{\mathrm{~B}(r, n-r+1)(k+1)}\left(\begin{array}{c}
n-r \\
j
\end{array}\right)\left(\begin{array}{c}
-j-r-1 \\
l
\end{array}\right)\left(\begin{array}{c}
j+r-1 \\
i
\end{array}\right)\left(\begin{array}{c}
\lambda(l+i+1)-1 \\
k
\end{array}\right) .
$$

Equation (38) reveals that the PDF of the TIHLIW order statistics is a mixture of IW densities. Hence, the qth moment of $X_{(r)}$ has the form

$$
E\left(X_{(r)}^{q}\right)=\sum_{k=0}^{\infty} b_{k}[\alpha(k+1)]^{(q / \beta)} \Gamma\left(1-\frac{q}{\beta}\right) .
$$

\section{Estimation Methods}

The estimation of the TIHLIW parameters is investigated using four methods of estimation, namely, the maximum likelihood estimators (MLEs), least squares estimators (LSEs), weighted least squares estimators (WLSEs), and Cramér-von Mises estimators (CVMEs). 
4.1. Maximum Likelihood. We determine the MLEs of the TIHLIW parameters. Let $\left(x_{1}, x_{2}, \ldots, x_{n}\right)$ be a random sample of size $n$ from TIHLIW $(\phi)$ where $\phi=(\lambda, \alpha, \beta)^{T}$. The log-likelihood function for $\phi$ has the form

$$
\begin{aligned}
\ell= & n \log (2 \lambda \alpha \beta) \\
& -(\beta+1) \sum_{i=1}^{n} \log x_{i}-\alpha \sum_{i=1}^{n} x_{i}^{\beta} \\
& +(\lambda-1) \sum_{i=1}^{n} \log \left(1-e^{-\alpha x_{i}^{-\beta}}\right) \\
& -2 \sum_{i=1}^{n} \log \left[1+\left(1-e^{-\alpha x_{i}^{-\beta}}\right)\right] .
\end{aligned}
$$

We can maximize the above log-likelihood equation by solving the nonlinear likelihood equations which follow by differentiating it. The associated components of the score vector

$$
U_{n}(\phi)=\left(\frac{\partial \ell}{\partial \lambda}, \frac{\partial \ell}{\partial \alpha}, \frac{\partial \ell}{\partial \beta}\right)^{T},
$$

are given by

$$
\begin{aligned}
& \frac{\partial \ell}{\partial \lambda}=\frac{n}{\lambda}+\sum_{i=1}^{n} \log \left(1-e^{-\alpha x_{i}^{-\beta}}\right) \\
& \frac{\partial \ell}{\partial \alpha}=\frac{n}{\alpha}-\sum_{i=1}^{n} x_{i}^{\beta}+(\lambda-1) \sum_{i=1}^{n} \frac{x_{i}^{-\beta} e^{-\alpha x_{i}^{-\beta}}}{1-e^{-\alpha x_{i}^{-\beta}}}-2 \sum_{i=1}^{n} \frac{x_{i}^{-\beta} e^{-\alpha x_{i}^{-\beta}}}{1+\left(1-e^{-\alpha x_{i}^{-\beta}}\right)} .
\end{aligned}
$$

And,

$$
\begin{aligned}
\frac{\partial \ell}{\partial \beta}= & \frac{n}{\beta}-\sum_{i=1}^{n} \log \left(x_{i}\right)-\alpha \sum_{i=1}^{n} x_{i}^{\beta} \log \left(x_{i}\right) \\
& +2 \sum_{i=1}^{n} \frac{\alpha \log \left(x_{i}\right) x_{i}^{-\beta} e^{-\alpha x_{i}^{-\beta}}}{1+\left(1-e^{-\alpha x_{i}^{-\beta}}\right)} \\
& +(\lambda-1) \sum_{i=1}^{n} \frac{\alpha \log \left(x_{i}\right) x_{i}^{-\beta}\left(e^{-\alpha x_{i}^{-\beta}}\right)}{1-e^{-\alpha x_{i}^{-\beta}}} .
\end{aligned}
$$

The MLEs of $\phi$ can be constructed by solving the nonlinear system $U_{n}(\phi)=0$ that cannot be analytically solved; hence, statistical programs are utilized to solve them numerically via iterative techniques such as a Newton-Raphson algorithm.

4.2. Ordinary and Weighted Least Squares. The least square and weighted least square methods are used to estimate the parameters of beta distribution [20]. Let $x_{(1)}<x_{(2)}<\cdots<x_{(n)}$ be the order statistics of a sample from the TIHLIW distribution, and then the LSEs and WLSEs of $\alpha, \lambda$, and $\beta$ can be obtained by minimizing the following function with respect to $\alpha, \lambda$, and $\beta$ :

$$
S(\alpha, \lambda, \beta)=\sum_{i=1}^{n} A_{i}\left\{\frac{1-\left[1-e^{-\alpha x_{(i)}^{-\beta}}\right]^{\lambda}}{1+\left[1-e^{-\alpha x_{(i)}^{-\beta}}\right]^{\lambda}}-\frac{i}{n+1}\right\}^{2}
$$

where $\left(A_{i}=1\right)$ in the case of LSEs and $\left(A_{i}=\left((n+1)^{2}(n+\right.\right.$ $2)) /(i(n-i+1)))$ in the case of WLSEs.

Further, the LSEs and WLSEs of the TIHLIW parameters are also obtained by solving the following nonlinear equations simultaneously with respect to $\alpha, \lambda$, and $\beta$ :

$$
\begin{aligned}
& \frac{\partial S(\alpha, \lambda, \beta)}{\partial \alpha}=\sum_{i=1}^{n} A_{i}\left\{\frac{1-\left[1-e^{-\alpha x_{(i)}^{-\beta}}\right]^{\lambda}}{1+\left[1-e^{-\alpha x_{(i)}^{-\beta}}\right]^{\lambda}}-\frac{i}{n+1}\right\} \phi_{1 i}=0, \\
& \frac{\partial S(\alpha, \lambda, \beta)}{\partial \lambda}=\sum_{i=1}^{n} A_{i}\left\{\frac{1-\left[1-e^{-\alpha x_{(i)}^{-\beta}}\right]^{\lambda}}{1+\left[1-e^{-\alpha x_{(i)}^{-\beta}}\right]^{\lambda}}-\frac{i}{n+1}\right\} \phi_{2 i}=0, \\
& \frac{\partial S(\alpha, \lambda, \beta)}{\partial \beta}=\sum_{i=1}^{n} A_{i}\left\{\frac{1-\left[1-e^{-\alpha x_{(i)}^{-\beta}}\right]^{\lambda}}{1+\left[1-e^{-\alpha x_{(i)}^{-\beta}}\right]^{\lambda}}-\frac{i}{n+1}\right\} \phi_{3 i}=0,
\end{aligned}
$$

where

$$
\begin{aligned}
\phi_{1 i}= & \frac{-2 \lambda x_{(i)}^{-\beta} e^{-\alpha x_{(i)}^{-\beta}}\left[1-e^{-\alpha x_{(i)}^{-\beta}}\right]^{\lambda-1}}{\left\{1+\left[1-e^{-\alpha x_{(i)}^{-\beta}}\right]^{\lambda}\right\}^{2}}, \\
\phi_{2 i}= & \frac{2\left[1-e^{-\alpha x_{(i)}^{-\beta}}\right]^{2 \lambda} \log \left[1-e^{-\alpha x_{(i)}^{-\beta}}\right]}{\left\{1+\left[1-e^{-\alpha x_{(i)}^{-\beta}}\right]^{\lambda}\right\}^{2}}, \\
\phi_{3 i}= & \frac{2 \lambda \alpha x_{(i)}^{-\beta} e^{-\alpha x_{(i)}^{-\beta} \log \left(x_{(i)}\right)\left[1-e^{-\alpha x_{(i)}^{-\beta}}\right]^{\lambda-1}}}{\left\{1+\left[1-e^{-\alpha x_{(i)}^{-\beta}}\right]^{\lambda}\right\}^{2}} .
\end{aligned}
$$

4.3. Cramér-von Mises. Based on the Cramér-von Mises (CVM) method [21, 22], the CVMEs of the TIHLIW parameters can be constructed by minimizing

$$
C(\alpha, \lambda, \beta)=\frac{1}{12 n}+\sum_{i=1}^{n}\left\{\frac{1-\left[1-e^{-\alpha x_{(i)}^{-\beta}}\right]^{\lambda}}{1+\left[1-e^{-\alpha x_{(i)}^{-\beta}}\right]^{\lambda}}-\frac{2 i-1}{2 n}\right\}^{2}
$$

with respect to $\alpha, \lambda$, and $\beta$. Further, the CVMEs can also be obtained by solving the following nonlinear equations simultaneously with respect to $\alpha, \lambda$, and $\beta$ : 


$$
\begin{aligned}
& \frac{\partial C(\alpha, \lambda, \beta)}{\partial \alpha}=\sum_{i=1}^{n}\left\{\frac{1-\left[1-e^{-\alpha x_{(i)}^{-\beta}}\right]^{\lambda}}{1+\left[1-e^{-\alpha x_{(i)}^{-\beta}}\right]^{\lambda}}-\frac{2 i-1}{2 n}\right\} \phi_{1 i}=0, \\
& \frac{\partial C(\alpha, \lambda, \beta)}{\partial \lambda}=\sum_{i=1}^{n}\left\{\frac{1-\left[1-e^{-\alpha x_{(i)}^{-\beta}}\right]^{\lambda}}{1+\left[1-e^{-\alpha x_{(i)}^{-\beta}}\right]^{\lambda}}-\frac{2 i-1}{2 n}\right\} \phi_{2 i}=0, \\
& \frac{\partial C(\alpha, \lambda, \beta)}{\partial \beta}=\sum_{i=1}^{n}\left\{\frac{1-\left[1-e^{-\alpha x_{(i)}^{-\beta}}\right]^{\lambda}}{1+\left[1-e^{-\alpha x_{(i)}^{-\beta}}\right]^{\lambda}}-\frac{2 i-1}{2 n}\right\} \phi_{3 i}=0,
\end{aligned}
$$

where $\phi_{1 i}, \phi_{2 i}$, and $\phi_{3 i}$ are, respectively, defined in equations (47)-(49).

\section{Simulation Study}

In this section, we conduct a simulation study to compare the performance of the different estimators based on the mean square error criterion. We compare the performances of the MLEs, LSEs, WLSEs, and CVMEs based on the mean square errors (MSEs) for different sample sizes. Mathematica 9 is utilized to obtain simulation results from 10,000 replications. The mean values of the MLEs, LSEs, WLSEs, and CVMEs and their MSEs of the TIHLIW parameters by considering sample sizes $n=50,100$, and 200 , are given for different values of parameters $\alpha, \lambda$, and $\beta$. The mean values of the MLEs, LSEs, WLSEs, and CVMEs and MSEs of the TIHLIW parameters are listed in Tables 1-3.

From Tables 1-3, we conclude the following:

(1) The MSE of $\alpha, \lambda$, and $\beta$ for all estimation methods decreases as $n$ increases.

(2) Table 1 shows that the MLEs have the lowest MSE in most cases of $\alpha$ and $\lambda$. Also, the WLSEs have the least MSE in most cases of $\beta$ at $\alpha=0.5, \lambda=0.5$, and $\beta=0.5$.

(3) Table 2 shows that CVMEs get the least MSE in most situations of $\alpha$ and $\lambda$. Also, the WLSEs have the lowest MSE in most cases of $\beta$ at $\alpha=0.5, \lambda=1.5$, and $\beta=0.5$.

(4) Table 3 shows that CVMEs have the lowest MSE in most cases of $\alpha, \lambda$, and $\beta$ at $\alpha=0.5, \lambda=1.5$, and $\beta=1.5$.

\section{Data Analysis}

In this section, we present an application to a real data set to illustrate the performance and flexibility of the TIHLIW distribution. The data refer to relief times of a sample of 20 patients who receive an analgesic [23]. These data have been analyzed by Afify et al. [24] and Cordeiro et al. [25].
TABLE 1: The mean values and MSEs of the TIHLIW distribution.

\begin{tabular}{|c|c|c|c|c|}
\hline Parameters & MLEs & LSEs & WLSEs & CVMEs \\
\hline \multicolumn{5}{|l|}{$n=50$} \\
\hline$\alpha=0.5$ & $\begin{array}{c}0.552 \\
(0.211)\end{array}$ & $\begin{array}{c}0.474 \\
(0.110)\end{array}$ & $\begin{array}{c}0.499 \\
(0.114)\end{array}$ & $\begin{array}{c}0.466 \\
(0.104)\end{array}$ \\
\hline$\lambda=1.5$ & $\begin{array}{c}1.877 \\
(3.526)\end{array}$ & $\begin{array}{c}1.056 \\
(1.525)\end{array}$ & $\begin{array}{c}0.985 \\
(1.589)\end{array}$ & $\begin{array}{c}1.492 \\
(0.800)\end{array}$ \\
\hline$\beta=0.5$ & $\begin{array}{c}0.612 \\
(0.094) \\
\end{array}$ & $\begin{array}{c}0.568 \\
(0.033) \\
\end{array}$ & $\begin{array}{c}0.565 \\
(0.039) \\
\end{array}$ & $\begin{array}{c}0.594 \\
(0.042) \\
\end{array}$ \\
\hline \multicolumn{5}{|l|}{$n=100$} \\
\hline$\alpha=0.5$ & $\begin{array}{c}0.551 \\
(0.119)\end{array}$ & $\begin{array}{c}0.456 \\
(0.062)\end{array}$ & $0.489(0.07)$ & $\begin{array}{c}0.454 \\
(0.053)\end{array}$ \\
\hline$\lambda=1.5$ & $\begin{array}{c}1.737 \\
(1.128)\end{array}$ & $\begin{array}{c}1.431 \\
(0.453)\end{array}$ & $\begin{array}{c}1.523 \\
(0.515)\end{array}$ & $\begin{array}{c}1.423 \\
(0.403)\end{array}$ \\
\hline$\beta=0.5$ & $\begin{array}{c}0.542 \\
(0.032) \\
\end{array}$ & $\begin{array}{c}0.558 \\
(0.019)\end{array}$ & $\begin{array}{c}0.544 \\
(0.019)\end{array}$ & $\begin{array}{c}0.563 \\
(0.019) \\
\end{array}$ \\
\hline \multicolumn{5}{|l|}{$n=200$} \\
\hline$\alpha=0.5$ & $\begin{array}{c}0.516 \\
(0.051)\end{array}$ & $0.433(0.03)$ & $\begin{array}{c}0.459 \\
(0.031)\end{array}$ & $\begin{array}{c}0.437 \\
(0.029)\end{array}$ \\
\hline$\lambda=1.5$ & $\begin{array}{c}1.578 \\
(0.340)\end{array}$ & $\begin{array}{c}1.351 \\
(0.179)\end{array}$ & $\begin{array}{c}1.419 \\
(0.187)\end{array}$ & $\begin{array}{c}1.368 \\
(0.175)\end{array}$ \\
\hline$\beta=0.5$ & $\begin{array}{c}0.524 \\
(0.013)\end{array}$ & $\begin{array}{c}0.556 \\
(0.012)\end{array}$ & $\begin{array}{c}0.540 \\
(0.010)\end{array}$ & $\begin{array}{c}0.553 \\
(0.011)\end{array}$ \\
\hline
\end{tabular}

\begin{tabular}{lcccc}
\hline Parameters & MLEs & LSEs & WLSEs & CVMEs \\
\hline$n=50$ & & & & \\
$\alpha=0.5$ & 0.559 & 0.620 & 0.612 & 0.633 \\
& $(0.244)$ & $(0.223)$ & $(0.201)$ & $(0.240)$ \\
$\lambda=0.5$ & 0.577 & 0.654 & 0.626 & 0.659 \\
& $(0.269)$ & $(0.280)$ & $(0.189)$ & $(0.299)$ \\
$\beta=0.5$ & 0.646 & 0.540 & 0.540 & 0.567 \\
& $(0.122)$ & $(0.066)$ & $(0.062)$ & $(0.078)$ \\
\hline$n=100$ & & & & \\
$\alpha=0.5$ & 0.522 & 0.588 & 0.578 & 0.574 \\
& $(0.103)$ & $(0.117)$ & $(0.103)$ & $(0.128)$ \\
$\lambda=0.5$ & 0.521 & 0.593 & 0.575 & 0.58 \\
& $(0.061)$ & $(0.099)$ & $(0.071)$ & $(0.122)$ \\
$\beta=0.5$ & 0.574 & 0.521 & 0.518 & 0.543 \\
& $(0.048)$ & $(0.042)$ & $(0.033)$ & $(0.045)$ \\
\hline$n=200$ & & & & \\
$\alpha=0.5$ & 0.504 & 0.538 & 0.532 & 0.553 \\
& $(0.055)$ & $(0.072)$ & $(0.059)$ & $(0.072)$ \\
$\lambda=0.5$ & 0.509 & 0.546 & 0.535 & 0.555 \\
& $(0.029)$ & $(0.049)$ & $(0.034)$ & $(0.051)$ \\
$\beta=0.5$ & 0.539 & 0.522 & 0.516 & 0.515 \\
& $(0.022)$ & $(0.025)$ & $(0.018)$ & $(0.023)$ \\
\hline
\end{tabular}

TABLE 2: The mean values and MSEs of the TIHLIW distribution.

For these data, we compare the TIHLIW model with some rival models, namely, the beta generalized inverse Weibull geometric (BGIWGc) by Elbatal et al. [26], transmuted complementary Weibull geometric (TCWGc) by Afify et al. [27], beta transmuted Weibull (BTW) by Afify et al. [28], McDonald log-logistic (McLL) by Tahir et al. [29], beta Weibull (BW) by Lee et al. [30], McDonald Weibull $(\mathrm{McW})$ by Cordeiro et al. [31], exponentiated transmuted generalized Rayleigh (ETGR) by Afify et al. [32], and new modified Weibull (NMW) [33] distributions. The PDFs of these distributions are given (for $x>0$ ) by 
TABLE 3: The mean values and MSEs of the TIHLIW distribution.

\begin{tabular}{|c|c|c|c|c|}
\hline Parameters & MLEs & LSEs & WLSEs & CVMEs \\
\hline \multicolumn{5}{|l|}{$n=50$} \\
\hline$\alpha=0.5$ & $0.602(0.276)$ & $0.544(0.060)$ & $0.545(0.056)$ & $0.563(0.064)$ \\
\hline$\lambda=1.5$ & $2.126(5.703)$ & $1.656(0.641)$ & $1.652(0.469)$ & $1.704(0.627)$ \\
\hline$\beta=1.5$ & $1.786(0.764)$ & $1.498(0.106)$ & $1.510(0.116)$ & $1.508(0.095)$ \\
\hline \multicolumn{5}{|l|}{$n=100$} \\
\hline$\alpha=0.5$ & $0.555(0.128)$ & $0.539(0.021)$ & $0.552(0.026)$ & $0.527(0.017)$ \\
\hline$\lambda=1.5$ & $1.775(1.598)$ & $1.600(0.128)$ & $1.642(0.161)$ & $1.583(0.096)$ \\
\hline$\beta=1.5$ & $1.618(0.297)$ & $1.452(0.036)$ & $1.447(0.047)$ & $1.493(0.040)$ \\
\hline \multicolumn{5}{|l|}{$n=200$} \\
\hline$\alpha=0.5$ & $0.516(0.049)$ & $0.524(0.009)$ & $0.540(0.014)$ & $0.530(0.009)$ \\
\hline$\lambda=1.5$ & $1.583(0.346)$ & $1.562(0.042)$ & $1.608(0.082)$ & $1.577(0.045)$ \\
\hline$\beta=1.5$ & $1.567(0.113)$ & $1.470(0.017)$ & $1.456(0.025)$ & $1.467(0.016)$ \\
\hline
\end{tabular}

TABLE 4: Goodness-of-fit measures for relief times data.

\begin{tabular}{|c|c|c|c|c|c|c|}
\hline Model & $-2 \widehat{\ell}$ & AIC & CAIC & HQIC & $\mathrm{AD}$ & CVM \\
\hline TIHLIW & 30.752 & 36.752 & 38.252 & 37.335 & 0.1746 & 0.0319 \\
\hline BGIWGc & 31.662 & 43.662 & 50.124 & 44.828 & 0.2467 & 0.0434 \\
\hline BTW & 33.051 & 43.051 & 74.337 & 44.023 & 0.3977 & 0.0689 \\
\hline TCWGc & 33.607 & 41.609 & 44.274 & 42.385 & 0.4360 & 0.0725 \\
\hline McLL & 33.854 & 43.855 & 48.140 & 44.826 & 0.4620 & 0.0790 \\
\hline $\mathrm{McW}$ & 33.907 & 43.907 & 48.193 & 44.879 & 0.4693 & 0.0802 \\
\hline BW & 34.396 & 42.396 & 45.063 & 43.174 & 0.5132 & 0.0873 \\
\hline ETGR & 36.856 & 44.857 & 47.523 & 45.634 & 0.7929 & 0.1363 \\
\hline NMW & 41.173 & 51.173 & 55.459 & 52.145 & 1.0678 & 0.1759 \\
\hline
\end{tabular}

TABLE 5: MLEs and SEs for relief times data.

\begin{tabular}{|c|c|c|c|c|c|c|}
\hline \multirow{2}{*}{$\begin{array}{l}\text { Model } \\
\text { TIHLIW } \\
(\alpha, \beta, \lambda)\end{array}$} & \multicolumn{6}{|c|}{ MLEs } \\
\hline & $\begin{array}{c}5.317 \\
(2.583)\end{array}$ & $\begin{array}{c}5.361 \\
(3.712)\end{array}$ & $\begin{array}{c}0.799 \\
(0.789)\end{array}$ & & & \\
\hline BGIWGc & 19.18 & 20.59 & 1.434 & 9.848 & $39 \cdot 10^{-5}$ & 5.8015 \\
\hline$(\alpha, \gamma, \theta, p, a, b)$ & (33.03) & $(43.24)$ & $(0.837)$ & $(2.001)$ & $(63.25)$ & $(4.346)$ \\
\hline BTW & 5.618 & 0.531 & 53.34 & 3.568 & -0.771 & \\
\hline$(\alpha, \beta, a, b, \lambda)$ & (9.353) & $(0.184)$ & (111.4) & $(4.265)$ & $(3.894)$ & \\
\hline TCWGc & 43.66 & 5.127 & 0.282 & -0.271 & & \\
\hline$(\alpha, \beta, \gamma, \lambda)$ & $(45.45)$ & $(0.814)$ & $(0.042)$ & $(0.656)$ & & \\
\hline McLL & 0.881 & 2.070 & 19.22 & 32.03 & 1.926 & \\
\hline$(\alpha, \beta, a, b, c)$ & $(0.109)$ & $(3.693)$ & $(22.34)$ & (43.07) & $(5.165)$ & \\
\hline $\mathrm{McW}$ & 2.773 & 0.380 & 79.10 & 17.89 & 3.006 & \\
\hline$(\alpha, \beta, a, b, c)$ & $(6.380)$ & $(0.188)$ & (119.1) & $(39.51)$ & $(13.96)$ & \\
\hline BW & 0.831 & 0.612 & 29.94 & 11.63 & & \\
\hline$(\alpha, \beta, a, b)$ & $(0.954)$ & $(0.340)$ & $(40.41)$ & $(21.90)$ & & \\
\hline ETGR & 0.103 & 0.691 & -0.342 & 23.53 & & \\
\hline$(\alpha, \beta, \lambda, \delta)$ & $(0.436)$ & $(0.086)$ & $(1.971)$ & $(105.3)$ & & \\
\hline NMW & 0.121 & 2.783 & $8.2 \cdot 10^{-5}$ & 0.0003 & 2.787 & \\
\hline$(\alpha, \beta, \gamma, \delta, \theta)$ & $(0.056)$ & $(20.37)$ & $\left(1.5 \cdot 10^{-3}\right)$ & $(0.025)$ & $(0.428)$ & \\
\hline
\end{tabular}

BGIWGc: $\quad f(x)=\left((1-p)^{b} / B(a, b)\right) \alpha \theta \gamma(\alpha x)^{-\theta-1}$ $e^{-\gamma a(\alpha x)^{-\theta}}\left(1-e^{-\gamma(\alpha x)^{-\theta}}\right)^{b-1} \times\left[1-p\left(1-e^{-\gamma(\alpha x)^{-\theta}}\right)\right]^{-a-b}$, where $p \in(0,1), \alpha, \beta, \gamma, a>0$ and $b>0$.

BTW: $f(x)=\left(\beta \alpha^{\beta} / B(a, b)\right) x^{\beta-1}\left[1-\lambda+2 \lambda e^{-(\alpha x)^{\beta}}\right]\{[1-$ $\left.\left.e^{-(\alpha x)^{\beta}}\right] \quad\left[1+\lambda e^{-(\alpha x)^{\beta}}\right]\right\}^{a-1} \times e^{-(\alpha x)^{\beta}}\left\{1-\left[1-e^{-(\alpha x)^{\beta}}\right][1+\right.$ $\left.\left.\lambda e^{-(\alpha x)^{\beta}}\right]\right\}^{b-1}$, where $\alpha, \beta, a, b>0$ and $|\lambda| \leq 1$.
TCWGc: $\quad f(x)=\alpha \beta \gamma(\gamma y)^{\beta-1} e^{-(\gamma y)^{\beta}}[\alpha(1-\lambda)-(\alpha-$ $\left.\alpha \lambda-\lambda-1) e^{-(\gamma y)^{\beta}}\right] \times\left[\alpha+(1-\alpha) e^{-(\gamma y)^{\beta}}\right]^{-3}$, where $\alpha, \beta$, $\gamma>0$ and $|\lambda| \leq 1$.

McLL: $\quad f(x)=(\alpha c /(B(a / c, b) \beta))(x / \beta)^{a \alpha-1}[1+$ $\left.(x / \beta)^{\alpha}\right]^{-a-1}\left(1-\left\{1-\left[1+(x / \beta)^{\alpha}\right]^{-1}\right\}^{c}\right)$, where $\alpha, \beta$, $a, b>0$ and $c>0$. 


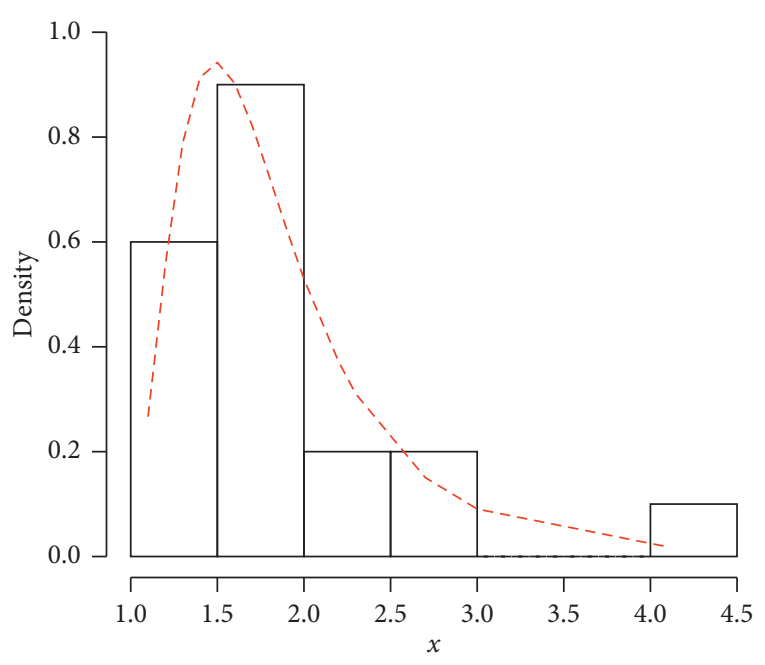

- - TIHLIW

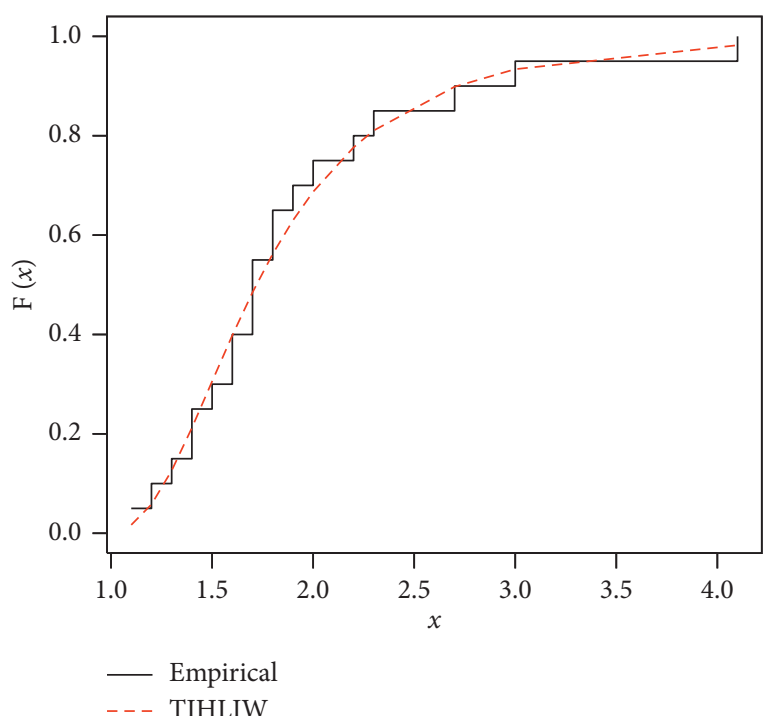

FIgURE 2: The fitted PDF and estimated CDF of the TIHLIW model.
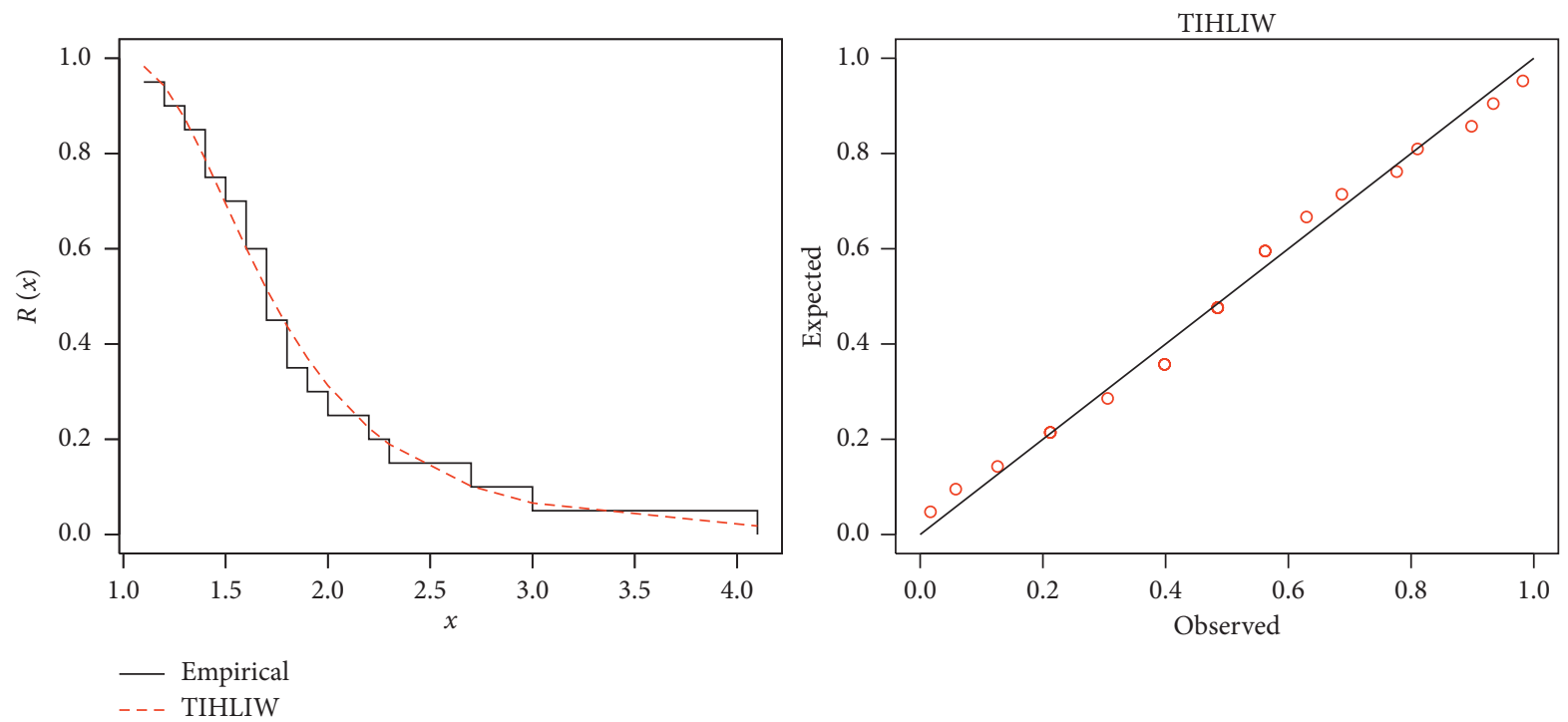

Figure 3: The estimated SF and PP plot of the TIHLIW model.

McW: $\quad f(x)=\left(\beta c \alpha^{\beta} /(B(a / c, b))\right) x^{\beta-1} e^{-(\alpha x)^{\beta}}[1-$ $\left.e^{-(\alpha x)^{\beta}}\right]^{a-1}\left\{1-\left[1-e^{-(\alpha x)^{\beta}}\right]^{c}\right\}^{b-1}$, where $\alpha, \beta, a, b>0$ and $c>0$.

BW: $f(x)=\left(\beta \alpha^{\beta} /(B(a, b))\right) x^{\beta-1} e^{-b(\alpha x)^{\beta}}[1-$ $\left.e^{-(\alpha x)^{\beta}}\right]^{a-1}$, where $\alpha, \beta, a>0$ and $b>0$.

ETGR: $\quad f(x)=2 \alpha \delta \beta^{2} x e^{-(\beta x)^{2}}\left[1-e^{-(\beta x)^{2}}\right]^{\alpha \delta-1} \times\{1+$ $\left.\lambda-2 \lambda\left[1-e^{-(\beta x)^{2}}\right]^{\alpha}\right\}\left\{1+\lambda-\lambda\left[1-e^{-(\beta x)^{2}}\right]^{\alpha}\right\}^{\alpha-1}$, where $\alpha, \beta, \delta>0$ and $|\lambda| \leq 1$.

NMW: $\quad f(x)=\left[\alpha \theta x^{\theta-1}+\gamma(\beta+\delta x) x^{\beta-1} e^{\delta x}\right]$ $e^{-\left(\alpha x^{\theta}+\gamma x^{\beta} e^{\delta x}\right)}$, where $\alpha, \beta, \theta, \gamma>0$ and $\delta>0$.

We consider some criteria including $-2 \widehat{\ell}$ (where $\widehat{\ell}$ is the maximized log-likelihood), AIC (Akaike information criterion), HQIC (Hannan-Quinn information criterion), CAIC (corrected Akaike information Criterion), AD (Anderson-Darling statistic), and CVM (Cramér-von Mises statistic), where Table 4 lists the numerical values of $-2 \hat{\ell}$, AIC, CAIC, HQIC, $A^{*}$, and $W^{*}$ for all fitted models, whereas MLEs and their standard errors (SEs) (in parentheses) are given in Table 5. From Table 4, the TIHLIW has the lowest values for all goodness-of-fit measures, and hence it provides close fits to relief times data than other fitted models.

The fitted PDF, estimated CDF, estimated survival function (SF), and PP plots of the TIHLIW distribution are shown in Figures 2 and 3.

\section{Conclusions}

We proposed a three-parameter type I half-logistic inverse Weibull (TIHLIW) distribution as a new extension of the inverse Weibull model. The TIHLIW density is a linear combination of the inverse Weibull densities. Some explicit 
expressions for mathematical quantities of the TIHLIW distribution are derived. We consider four methods of estimation, namely, the maximum likelihood, least squares, weighted least squares, and Cramér-von Mises methods, to estimate the TIHLIW parameters. The performance of these proposed estimation methods is conducted via some simulations. A real data application proves that the TIHLIW model provides consistently better fits compared to some other rival models.

\section{Data Availability}

This work is mainly a methodological development and has been applied on secondary data related to the relief times of 20 patients who received an analgesic, but if required, data will be provided.

\section{Conflicts of Interest}

The authors declare that there are no conflicts of interest regarding the publication of this paper.

\section{Acknowledgments}

The authors are grateful to the Deanship of Scientific Research at King Saud University represented by the Research Center at the College of Business for financially supporting this research.

\section{References}

[1] A. Drapella, "The complementary weibull distribution: Unknown or just forgotten?" Quality and Reliability Engineering International, vol. 9, no. 4, pp. 383-385, 1993.

[2] G. S. Mudholkar and G. D. Kollia, "Generalized Weibull family: A structural analysis," Communications in Statistics Theory and Methods, vol. 23, no. 4, pp. 1149-1171, 1994.

[3] A. Z. Keller, A. R. R. Kamath, and U. D. Perera, "Reliability analysis of CNC machine tools," Reliability Engineering, vol. 3, no. 6, pp. 449-473, 1982.

[4] M. S. Khan and R. King, "Modified inverse Weibull distribution," Journal of Statistics Applications \& Probability, vol. 1, no. 2, pp. 115-132, 2012.

[5] M. S. Khan, "The beta inverse Weibull distribution," International Transactions in Mathematical Sciences and Computer, vol. 3, pp. 113-119, 2010.

[6] F. R. S. de Gusmão, E. M. M. Ortega, and G. M. Cordeiro, "The generalized inverse Weibull distribution," Statistical Papers, vol. 52, no. 3, pp. 591-619, 2011.

[7] M. Pararai, G. Warahena-Liyanage, and B. O. Oluyede, "A new class of generalized inverse Weibull distribution with applications," Journal of Applied Mathematics and Bioinformatics, vol. 4, pp. 17-35, 2014.

[8] B. O. Oluyede and T. Yang, "Generalizations of the inverse Weibull and related distributions with applications," Electronic Journal of Applied Statistical Analysis, vol. 7, pp. 94116, 2014.

[9] G. Aryal and I. Elbatal, "Kumaraswamy modified inverse Weibull distribution: Theory and application," Applied Mathematics \& Information Sciences, vol. 9, no. 2, pp. 651660, 2015.
[10] H. M. Okasha, A. H. El-Baz, A. M. K. Tarabia, and A. M. Basheer, "Extended inverse Weibull distribution with reliability application," Journal of the Egyptian Mathematical Society, vol. 25, no. 3, pp. 343-349, 2017.

[11] A. M. Basheer, "Alpha power inverse Weibull distribution with reliability application," Journal of Taibah University for Science, vol. 13, no. 1, pp. 423-432, 2019.

[12] A. Marshall and I. Olkin, "A new method for adding a parameter to a family of distributions with application to the exponential and Weibull families," Biometrika, vol. 84, no. 3, pp. 641-652, 1997.

[13] W. T. Shaw and I. R. C. Buckley, "The alchemy of probability distributions: Beyond Gram-Charlier expansions and a SkewKurtotic-Normal distribution from a rank transmutation map," 2007, http://arxiv.org/abs/0901.0434.

[14] G. M. Cordeiro and M. de Castro, "A new family of generalized distributions," Journal of Statistical Computation and Simulation, vol. 81, no. 7, pp. 883-898, 2011.

[15] M. Alizadeh, E. Altun, A. Z. Afify, and G. Ozel, "The extended odd Weibull-G family: Properties and applications," Communications Faculty of Science University of Ankara Series A1Mathematics and Statistics, vol. 68, no. 1, pp. 161-186, 2018.

[16] G. M. Cordeiro, A. Z. Afify, E. M. M. Ortega, A. K. Suzuki, and M. E. Mead, "The odd Lomax generator of distributions: Properties, estimation and applications," Journal of Computational and Applied Mathematics, vol. 347, pp. 222-237, 2019.

[17] G. M. Cordeiro, M. Alizadeh, and P. R. Diniz Marinho, "The type I half-logistic family of distributions," Journal of Statistical Computation and Simulation, vol. 86, no. 4, pp. 707728, 2016.

[18] E. M. Wright, "The asymptotic expansion of the generalized hypergeometric function," Journal of the London Mathematical Society, vol. 10, no. 4, pp. 286-293, 1935.

[19] C. D. Lai and M. Xie, Stochastic Ageing and Dependence for Reliability, Springer Science and Business Media, Berlin, Germany, 2006.

[20] J. J. Swain, S. Venkatraman, and J. R. Wilson, "Least-squares estimation of distribution functions in johnson's translation system," Journal of Statistical Computation and Simulation, vol. 29, no. 4, pp. 271-297, 1988.

[21] H. Cramér, "On the composition of elementary errors," Scandinavian Actuarial Journal, vol. 1928, no. 1, pp. 13-74, 1928.

[22] R. E. Von Mises, Wahrs cheinlichkeit, Statistik und Wahrheit, Springer, Berlin, Germany, 1928.

[23] A. J. Gross and V. A. Clark, Survival Distributions: Reliability Applications in the Biomedical Sciences, John Wiley \& Sons, Hoboken, NJ, USA, 1975.

[24] A. Z. Afify, E. Altun, M. Alizadeh, G. Ozel, and G. G. Hamedani, "The odd exponentiated half-logistic-G family: properties, characterizations and applications," Chilean Journal of Statistics, vol. 8, pp. 65-91, 2017.

[25] G. Cordeiro, M. Mead, A. Z. Afify, A. Suzuki, and A. Abd ElGaied, "An extended Burr XII distribution: Properties, inference and applications," Pakistan Journal of Statistics and Operation Research, vol. 13, no. 4, pp. 809-828, 2017a.

[26] I. Elbatal, Y. M. El Gebaly, and E. A. Amin, "The beta generalized inverse Weibull geometric distribution," Pakistan Journal of Statistics and Operation Research, vol. 13, no. 1, pp. 75-90, 2017.

[27] A. Z. Afify, Z. M. Nofal, and N. S. Butt, "Transmuted complementary Weibull geometric distribution," Pakistan Journal 
of Statistics and Operations Research, vol. 10, pp. 435-454, 2014.

[28] A. Afify, H. Yousof, and S. Nadarajah, "The beta transmuted$\mathrm{H}$ family for lifetime data," Statistics and Its Interface, vol. 10, no. 3, pp. 505-520, 2017.

[29] M. H. Tahir, M. Mansoor, M. Zubair, and G. G. Hamedani, "McDonald log-logistic distribution with an application to breast cancer data," Journal of Statistical Theory and Applications, vol. 13, no. 1, pp. 65-82, 2014.

[30] C. Lee, F. Famoye, and O. Olumolade, "Beta-Weibull distribution: some properties and applications to censored data," Journal of Modern Applied Statistical Methods, vol. 6, no. 1, pp. 173-186, 2007.

[31] G. M. Cordeiro, E. M. Hashimoto, and E. M. M. Ortega, "The McDonald Weibull model," Statistics, vol. 48, no. 2, pp. 256-278, 2014.

[32] A. Z. A Afify, Z. M. Nofal, and A. E. H. N. Ebraheim, "Exponentiated transmuted generalized Rayleigh distribution: A new four parameter Rayleigh distribution," Pakistan Journal of Statistics and Operations Research, vol. 11, no. 1, pp. 115-134, 2015.

[33] S. J. Almalki and J. Yuan, "A new modified Weibull distribution," Reliability Engineering \& System Safety, vol. 111, pp. 164-170, 2013. 